MATHEMATICS OF COMPUTATION

Volume 81, Number 278, April 2012, Pages 1149-1161

S 0025-5718(2011)02547-0

Article electronically published on September 30, 2011

\title{
EXPLICIT COMPUTATIONS ON THE DESINGULARIZED KUMMER SURFACE
}

\author{
V. G. LOPEZ NEUMANN AND CONSTANTIN MANOIL
}

\begin{abstract}
We find formulas for the birational maps from a Kummer surface $\mathcal{K}$ and its dual $\mathcal{K}^{*}$ to their common minimal desingularization $\mathcal{S}$. We show how the nodes of $\mathcal{K}$ and $\mathcal{K}^{*}$ blow up. Then we give a description of the group of linear automorphisms of $\mathcal{S}$.
\end{abstract}

\section{INTRODUCTION}

In the 19th century a singular surface $\mathcal{K}$, called the Kummer surface, was attached to a quadratic line complex. A minimal desingularization $\Sigma$ of $\mathcal{K}$ and a birational map $\kappa_{1}: \mathcal{K} \rightarrow \Sigma$ were constructed by geometric methods. One may call this the classical construction of the Kummer surface, which we recall in Section 4.

Another construction is the following: Let $A$ be an abelian surface, and let $\sigma$ be the involution of $A$ given by $\sigma(x)=-x$. The quotient $\mathcal{K}=A / \sigma$ has 16 double points, and one defines a K3 surface $\mathcal{S}$ to be $\mathcal{K}$ with these 16 nodes blown up ([1], Prop. 8.11). To be consistent with the historical point of view and with our main reference [3], we call $\mathcal{S}$ the desingularized Kummer surface. If $A=\mathcal{J}(\mathcal{C})$, where $\mathcal{J}(\mathcal{C})$ is the Jacobian of a curve $\mathcal{C}$ of genus 2, then $\mathcal{K}$ is called the Kummer surface belonging to $\mathcal{C}$. The connection between the two constructions of the Kummer surface is explicitly established in [3], Chapter 17 (see Lemma 4.5).

A desingularization $\mathcal{S}$ of $\mathcal{K}$ is constructed explicitly in [3], Chapter 16, by algebraic methods. Denote by $\mathcal{K}^{*}$ the projective dual of $\mathcal{K}$. There are birational maps $\kappa: \mathcal{K} \rightarrow \mathcal{S}$ and $\kappa^{*}: \mathcal{K}^{*} \rightarrow \mathcal{S}$ and morphisms extending $\kappa^{-1}: \mathcal{S} \rightarrow \mathcal{K}$ and $\kappa^{*-1}: \mathcal{S} \rightarrow \mathcal{K}^{*}$ to all of $\mathcal{S}$. We denote these extensions also by $\kappa^{-1}$ and $\kappa^{*-1}$. They are minimal desingularizations of $\mathcal{K}$ and $\mathcal{K}^{*}$.

Origins. Cassels and Flynn explain that the surface $\mathcal{S}$ comes from the behavior of six of the tropes (see Definition 2.3) under the duplication map. The existence of $\mathcal{S}$ raises more far-reaching questions. Indeed, if the ground field $k$ is algebraically closed, one has a commutative diagram:

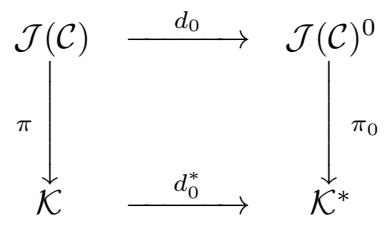

Received by the editor July 3, 2009.

2010 Mathematics Subject Classification. Primary 14J28, 14M15; Secondary 14J50.

Key words and phrases. Genus 2 curves, Kummer surfaces, line complexes.

(C)2011 American Mathematical Society Reverts to public domain 28 years from publication 
where $\mathcal{J}(\mathcal{C})^{0}$ is the dual of $\mathcal{J}(\mathcal{C})$ as an abelian variety. Here, the maps $d_{0}$ and $d_{0}^{*}$ depend on the choice of a rational point on $\mathcal{C}$. Thus the abelian varieties duality matches with the projective one. When $k$ is not algebraically closed, one has to enlarge the ground field to obtain such diagrams, yet $\mathcal{S}$ is a desingularization over $k$ of both $\mathcal{K}$ and $\mathcal{K}^{*}$. One may ask if there is a unifying object for $\mathcal{J}(\mathcal{C})$ and $\mathcal{J}(\mathcal{C})^{0}$, generalizing the abelian varieties duality.

Recent developments. The Jacobian $\mathcal{J}(\mathcal{C})$ can be embedded in $\mathbb{P}^{15}$ and is described by 72 quadratic equations (4). More computable objects, $\mathcal{S}$ and its twists, appeared in recent attempts by M. Stoll and N. Bruin, to compute the Mordell-Weil group of $\mathcal{J}(\mathcal{C})$. We give a brief account of it in Section 5 of 8 .

Cassels and Flynn already suggested that the 2-Selmer group could be investigated by using twists of $\mathcal{S}$. In 2007 A. Logan and R. van Luijk ([7]) and P. Corn ([2]) made use of twists of $\mathcal{S}$ to find specific curves with nontrivial 2-torsion elements in the Tate-Shafarevich groups of their Jacobians.

Our results and structure of this paper. In Section 2 we give a background. The rare relevant facts, not included in this paper, are contained in [3] and 8 .

This paper is structured along two computational ideas. First, to profit from the algebraic construction of $\mathcal{S}$ in 3 in order to describe its linear automorphism. Second, to link $\mathcal{S}$ and $\Sigma$ and thus bring line complexes into the picture. Our results achieve part of the program suggested in $\underline{3}$, at the end of Chapter 16.

In Section 3 we complete the construction in [3], enlarge the set of points where $\kappa: \mathcal{K} \rightarrow \mathcal{S}$ is explicitly defined and find formulas for $\kappa$. These formulas allow one to describe how each singularity of $\mathcal{K}$ and $\mathcal{K}^{*}$ blows up (see Lemma 3.6).

In Section 4 we define an explicit isomorphism $\Theta$ between $\Sigma$ and $\mathcal{S}$. We observe that well-known projective isomorphisms $W_{i}: \mathcal{K} \longrightarrow \mathcal{K}^{*}$ lift to $\Sigma$ to correspondences given by line complexes of degree 1 . Using $\Theta$ we show that the $W_{i}$ lift to $\mathcal{S}$ to commuting involutions $\varepsilon^{(i)}$ (Corollary 4.12). With the formulas for $\kappa$ at hand, one can now find formulas for $\kappa^{*}$.

In Section 5 we give a description of the group of linear automorphisms of $\mathcal{S}$.

\section{Preliminaries}

We work over a field $k$ with $\operatorname{char}(k) \neq 2$ and with more than 5 elements. By a curve, we mean a smooth projective irreducible variety of dimension 1 . Throughout this paper, $\mathcal{C}$ will be a curve of genus 2 . Such a curve admits an affine model:

$$
\mathcal{C}^{\prime}: \quad Y^{2}=F(X)=f_{0}+f_{1} X+\cdots+f_{6} X^{6} \in k[X], \quad f_{6} \neq 0
$$

and $F$ has distinct roots $\theta_{1}, \ldots, \theta_{6}$. The points $\mathfrak{a}_{i}=\left(\theta_{i}, 0\right)$ are the Weierstrass points. We denote by $\infty^{ \pm}$the points at infinity on the completion $\mathcal{C}$ of $\mathcal{C}^{\prime}$. For a given point $\mathfrak{r}=(x, y)$ on $\mathcal{C}$, the conjugate of $\mathfrak{r}$ under the $\pm Y$ involution is the point $\overline{\mathfrak{r}}=(x,-y)$. For a divisor $\mathfrak{X}=\sum n_{i} \mathfrak{r}_{i}$ we denote by $\overline{\mathfrak{X}}=\sum n_{i} \overline{\mathfrak{r}}_{i}$. The class of a divisor $\mathfrak{X}$ is denoted by $[\mathfrak{X}]$ and a divisor in the canonical class by $K_{\mathcal{C}}$.

One can regard a point of the Jacobian $J(\mathcal{C})$ as the class of a divisor $\mathfrak{X}=\mathfrak{r}+\mathfrak{u}$, where $\mathfrak{r}=(x, y), \mathfrak{u}=(u, v)$ is a pair of points on $\mathcal{C}$. Starting from this, Flynn constructs a projective embedding of the Jacobian as follows (4]). For a point $\mathfrak{X}=$ $\{\mathfrak{r}, \mathfrak{u}\}$ on $\mathcal{C}^{(2)}$ (symmetric product) with $\mathfrak{r}=(x, y), \mathfrak{u}=(u, v)$, define:

$$
\sigma_{0}=1, \quad \sigma_{1}=x+u, \quad \sigma_{2}=x u, \quad \beta_{0}=\frac{F_{0}(x, u)-2 y v}{(x-u)^{2}},
$$


where

$$
\begin{aligned}
F_{0}(x, u)= & 2 f_{0}+f_{1}(x+u)+2 f_{2} x u+f_{3} x u(x+u) \\
& +2 f_{4}(x u)^{2}+f_{5}(x u)^{2}(x+u)+2 f_{6}(x u)^{3} .
\end{aligned}
$$

The Jacobian is then the projective locus of $\mathbf{z}=\left(z_{0}: \ldots: z_{15}\right)$ in $\mathbb{P}^{15}$, where $z_{0}=$ $\delta ; z_{1}=\gamma_{1} ; z_{2}=\gamma_{0} ; z_{i}=\beta_{5-i}, i=3,4,5 ; z_{i}=\alpha_{9-i}, i=6, \ldots, 9 ; z_{i}=\sigma_{14-i}$, $i=10, \ldots, 14$; and $z_{15}=\rho$. For the definition of the functions $\alpha, \beta$, etc. and details, see [3], Chapter 2 .

Definition 2.1. The Kummer surface $\mathcal{K}$ belonging to a curve of genus 2 , is the projective locus in $\mathbb{P}^{3}$ of the elements $\xi=\left(\xi_{1}: \xi_{2}: \xi_{3}: \xi_{4}\right)=\left(\sigma_{0}: \sigma_{1}: \sigma_{2}: \beta_{0}\right)$.

The equation of the Kummer surface is

$$
\mathcal{K}: \quad K=K_{2} \xi_{4}^{2}+K_{1} \xi_{4}+K_{0}=0,
$$

where the $K_{i}$ are forms of degree $4-i$ in $\xi_{1}, \xi_{2}, \xi_{3}$ ([3], formula (3.1.9)). The natural map from $J(\mathcal{C})$ to $\mathcal{K}$ given by

$$
\left(z_{0}: \ldots: z_{15}\right) \longmapsto\left(z_{14}: z_{13}: z_{12}: z_{5}\right)=\left(\xi_{1}: \ldots: \xi_{4}\right)
$$

is 2 to 1 ; the ramification points correspond to divisor classes $[\mathfrak{X}]$ with $[\mathfrak{X}]=[\overline{\mathfrak{X}}]$. The images of these classes are the 16 nodes on $\mathcal{K}: N_{0}=(0: 0: 0: 1)$ corresponds to $\left[K_{\mathcal{C}}\right]$ and 15 nodes $N_{i j}$ correspond to classes $\left[\mathfrak{X}_{i j}\right]=\left[\mathfrak{a}_{i}+\mathfrak{a}_{j}\right]$ with $i \neq j$.

Definition 2.2. The dual Kummer surface $\mathcal{K}^{*} \subset\left(\mathbb{P}^{3}\right)^{\checkmark}=\mathbb{P}^{3}$ is the projective dual of $\mathcal{K}$, i.e., to the point $\xi \in \mathcal{K}$ corresponds the point $\eta \in \mathcal{K}^{*}$ such that $\eta=\left(\eta_{1}: \eta_{2}\right.$ : $\left.\eta_{3}: \eta_{4}\right) \in\left(\mathbb{P}^{3}\right)^{\checkmark}$ gives the tangent plane to $\mathcal{K}$ at $\xi$.

Definition 2.3. There are 6 planes $T_{i}$ containing the 6 nodes $N_{0}$ and $N_{i j}, j \neq i$ and 10 planes $T_{i j k}$ containing the 6 nodes $N_{m n}$ for $\{m, n\} \subset\{i, j, k\}$ or $\{m, n\} \cap$ $\{i, j, k\}=\emptyset$. These are the tropes; they cut conics on $\mathcal{K}$. They correspond to the 16 singular points of $\mathcal{K}^{*}$. The equations of the $T_{i}$ are:

$$
T_{i}: \quad \theta_{i}^{2} \xi_{1}-\theta_{i} \xi_{2}+\xi_{3}=0
$$

Lemma 2.4. For each Weierstrass point $\mathfrak{a}_{i}=\left(\theta_{i}, 0\right)$ there is a projective map $W_{i}: \mathcal{K} \longrightarrow \mathcal{K}^{*}$ induced by the addition of $\mathfrak{a}_{i}$ to a divisor of degree 2 . One has $W_{i}\left(N_{0}\right)=T_{i}$ (singular point of $\mathcal{K}^{*}$ ). Furthermore, $W_{i}^{-1} \circ W_{j}\left(N_{0}\right)=N_{i j}$.

Proof. See [3, Lemma 4.5.1.

\section{The Desingularized Kummer surface}

We recall the facts from [3], Chapter 16 we need, keeping the notation there.

Let $\mathbf{p}=\left(p_{0}: \ldots: p_{5}\right)$, where the $p_{j}$ are indeterminates, and put $P(X)=$ $\sum_{0}^{5} p_{j} X^{j}$. Let $\mathcal{S}$ be the projective locus of the $\mathbf{p}$ for which $P(X)^{2}$ is congruent to a quadratic in $X$ modulo $F(X)$. Put

$$
P_{j}(X)=\prod_{i \neq j}\left(X-\theta_{i}\right)=\sum_{k=0}^{5} h_{j k} X^{k}
$$

and $\omega_{j}=P_{j}\left(\theta_{j}\right) \neq 0$. Since $\theta_{i} \neq \theta_{j}$ for $i \neq j$, we have $\omega_{j} \neq 0$ and the $P_{j}$ span the vector space of polynomials of degree at most 5 . We have

$$
P(X)=\sum_{j} \pi_{j} P_{j}(X), \quad \text { where } \quad \pi_{j}=\frac{P\left(\theta_{j}\right)}{\omega_{j}} .
$$


The $K 3$ surface $\mathcal{S}$ is the complete intersection in $\mathbb{P}^{5}$ of the quadrics $\mathcal{S}_{0}, \mathcal{S}_{1}, \mathcal{S}_{2}$ where

$$
\mathcal{S}_{i}: S_{i}=0 \quad \text { and } \quad S_{i}=\sum_{j} \theta_{j}^{i} \omega_{j} \pi_{j}^{2} \quad \text { for } \quad i=0,1,2
$$

It is a minimal desingularization of $\mathcal{K}$ and of $\mathcal{K}^{*}$. Here the $S_{i}$ are quadratic forms in $\mathbf{p}$ with coefficients in $\mathbb{Z}\left[f_{1}, \ldots, f_{6}\right]$.

The following theorems hold ([3], Theorems 16.5.1 and 16.5.3):

Theorem 3.1. There is a birational map $\kappa: \mathcal{K} \rightarrow \mathcal{S}$ defined for general $\xi \in \mathcal{K}$ as follows: Let $\mathfrak{X}=\{(x, y),(u, v)\}$ correspond to $\xi$. Put $G(X)=(X-x)(X-u)$ and let $M(X)$ be the cubic determined by the property that $Y-M(X)$ vanishes twice on $\mathfrak{X}$. Let $P(X)=\sum_{0}^{5} p_{j} X^{j}$ be determined by $G P \equiv M \bmod F$. Then $\kappa(\xi)$ is the point with projective coordinates $\left(p_{0}: \ldots: p_{5}\right)$.

Let $\kappa^{*}: \mathcal{K}^{*} \rightarrow \mathcal{S}$ be the birational map defined in [3], Theorem 16.5.2.

Theorem 3.2. Let $\xi \in \mathcal{K}$ and $\eta \in \mathcal{K}^{*}$ be dual, that is, $\eta$ gives the tangent to $\mathcal{K}$ at $\xi$. Then $\kappa(\xi)=\kappa^{*}(\eta)$.

Our first result is the following.

Lemma 3.3. The map $\kappa: \mathcal{K} \rightarrow \mathcal{S}$ from Theorem 3.1 is given by the formulas listed below.

Proof. The problem is to make effective the method given in [3], Chapter 16. For completeness and due to typing errors there, we recall it in [8, in the Appendix. As presented in $[3]$, the method works for a general element $[\mathfrak{X}]=[(x, y)+(u, v)]$, where $y v \neq 0$ and $x \neq u$.

First, we put $y^{2}=F(x), v^{2}=F(u), y v=\left[F_{0}(x, u)-\beta_{0}(x-u)^{2}\right] / 2$ in the coefficients of $P(X)$, then as the resulting coefficients are symmetric functions of $x$ and $u$, we express them in terms of $\xi_{2}=x+u$ and $\xi_{3}=x u$. Finally, we homogenize the formulas with respect to $\xi_{1}=1, \xi_{2}, \xi_{3}, \xi_{4}$. One first obtains

$$
\kappa(\xi)=\left(\tilde{p}_{0}(\xi): \ldots: \tilde{p}_{5}(\xi)\right)
$$

where

$$
\tilde{p}_{j}(\xi)=\alpha_{j} K_{2}+\beta_{j}\left(K_{1} \xi_{4}+K_{0}\right), \quad \text { for } 0 \leq j \leq 5 .
$$

Here $\alpha_{j}$ and $\beta_{j}$ are homogeneous forms in $\xi$ of degree 4 and 2, respectively, and the $K_{j}$ are those in (2.2). Taking $p_{j}(\xi)=\left(\tilde{p}_{j}(\xi)-\beta_{j} K\right) / K_{2}=\alpha_{j}-\beta_{j} \xi_{4}^{2}$, we obtain formulas of degree 4 for $\kappa$, which will be defined also for $K_{2}=0$, extending $\kappa$ to images of divisor classes $[\mathfrak{X}]=[(x, y)+(u, v)]$ with $x=u$ and $y=v \neq 0$. However, the formulas do not work for points with $F^{\prime}(x)=0$ and for the image of $\left[2 \infty^{+}\right]$. We will treat the case $y=0$ or $v=0$ in connection with nodes and tropes. 


\section{Polynomial definition of $\kappa$.}

$$
\begin{aligned}
& p_{0}=-f_{3} f_{6} \xi_{1} \xi_{3}^{3}+1 / 2 f_{5}^{2} \xi_{2} \xi_{3}^{3}-2 \xi_{3}^{3} f_{4} f_{6} \xi_{2}+2 \xi_{3}^{2} \xi_{1}^{2} f_{1} f_{6}-\xi_{3}^{2} \xi_{1}^{2} f_{5} f_{2} \\
& -2 \xi_{3}^{2} \xi_{1} \xi_{2} f_{6} f_{2}-1 / 2 \xi_{3}^{2} \xi_{1} f_{5} \xi_{4}-1 / 2 \xi_{3}^{2} \xi_{1} \xi_{2} f_{5} f_{3}-\xi_{3}^{2} \xi_{2}^{2} f_{6} f_{3}-2 \xi_{3}^{2} \xi_{2} f_{6} \xi_{4} \\
& -1 / 2 \xi_{3} f_{3} \xi_{4} \xi_{1}^{2}-3 / 2 \xi_{3} \xi_{1}^{2} \xi_{2} f_{5} f_{1}-\xi_{3} \xi_{2} f_{4} \xi_{4} \xi_{1}-3 \xi_{3} \xi_{1} \xi_{2}^{2} f_{6} f_{1} \\
& -1 / 2 \xi_{3} \xi_{2}^{2} f_{5} \xi_{4}+f_{1} f_{2} \xi_{1}^{4}+\xi_{1}^{3} \xi_{2} f_{1} f_{3}+3 / 2 \xi_{1}^{3} f_{1} \xi_{4}+\xi_{2}^{2} f_{4} f_{1} \xi_{1}^{2}+\xi_{2}^{3} f_{5} f_{1} \xi_{1} \\
& +\xi_{2}^{4} f_{1} f_{6}-1 / 2 \xi_{1} \xi_{2} \xi_{4}^{2} \\
& p_{1}=2 \xi_{1}^{4} f_{2}^{2}-2 \xi_{3} \xi_{1} \xi_{2}^{2} f_{6} f_{2}+1 / 2 \xi_{1}^{2} \xi_{2}^{2} f_{5} f_{1}-1 / 2 \xi_{1}^{4} f_{3} f_{1}+2 \xi_{2}^{4} f_{2} f_{6}+3 \xi_{1}^{3} \xi_{4} f_{2} \\
& +1 / 2 \xi_{3} f_{3}^{2} \xi_{1}^{3}+1 / 2 \xi_{2}^{3} f_{5} \xi_{4}+\xi_{3} \xi_{1}^{2} \xi_{2} f_{4} f_{3}-1 / 2 \xi_{3}^{2} \xi_{2}^{2} f_{5}^{2}+3 / 2 \xi_{3} \xi_{1} \xi_{2}^{2} f_{5} f_{3} \\
& +2 \xi_{3}^{2} f_{4} f_{6} \xi_{2}^{2}-\xi_{3} \xi_{1}^{2} \xi_{2} f_{5} f_{2}+\xi_{3} \xi_{2}^{2} f_{6} \xi_{4}+2 \xi_{3} \xi_{2}^{3} f_{6} f_{3}+\xi_{1}^{2} \xi_{4}^{2}+2 \xi_{1}^{3} \xi_{2} f_{2} f_{3} \\
& -\xi_{3} \xi_{1}^{2} \xi_{2} f_{6} f_{1}+2 \xi_{1}^{2} f_{2} f_{4} \xi_{2}^{2}+3 / 2 \xi_{1}^{2} \xi_{2} f_{3} \xi_{4}+\xi_{1} f_{4} \xi_{4} \xi_{2}^{2}+\xi_{1} \xi_{2}^{3} f_{6} f_{1}+2 \xi_{1} \xi_{2}^{3} f_{5} f_{2} \\
& +2 \xi_{3}^{2} \xi_{1}^{2} f_{6} f_{2}-1 / 2 \xi_{3}^{2} \xi_{1}^{2} f_{5} f_{3}+\xi_{3}^{2} \xi_{4} f_{6} \xi_{1} \\
& p_{2}=2 \xi_{1}^{2} \xi_{2}^{2} f_{4} f_{3}-f_{6} f_{5} \xi_{1} \xi_{3}^{3}+\xi_{3}^{2} \xi_{1}^{2} f_{3} f_{6}-\xi_{3}^{2} \xi_{1}^{2} f_{4} f_{5}+\xi_{3}^{2} \xi_{1} f_{5}^{2} \xi_{2} \\
& +\xi_{3} f_{1} f_{6} \xi_{1}^{3}+\xi_{3} \xi_{1}^{3} f_{3} f_{4}-2 \xi_{3} f_{5} f_{2} \xi_{1}^{3}+2 \xi_{3} \xi_{1}^{2} \xi_{2} f_{4}^{2}-2 \xi_{3} f_{5} \xi_{4} \xi_{1}^{2} \\
& -\xi_{1}^{4} f_{1} f_{4}+2 \xi_{1}^{4} f_{3} f_{2}-2 \xi_{3} \xi_{2} f_{6} f_{2} \xi_{1}^{2}-5 \xi_{3} \xi_{2}^{2} f_{6} f_{3} \xi_{1}+2 \xi_{3} \xi_{1} \xi_{2}^{2} f_{5} f_{4} \\
& -3 \xi_{3} f_{6} \xi_{4} \xi_{2} \xi_{1}-3 \xi_{3} \xi_{2} f_{5} f_{3} \xi_{1}^{2}+2 \xi_{2}^{4} f_{3} f_{6}+\xi_{2}^{3} f_{6} \xi_{4}+2 f_{3} \xi_{4} \xi_{1}^{3}+2 \xi_{2} f_{3}^{2} \xi_{1}^{3} \\
& +2 \xi_{2}^{3} f_{5} f_{3} \xi_{1}-\xi_{2} f_{5} f_{1} \xi_{1}^{3}-\xi_{2}^{2} f_{6} f_{1} \xi_{1}^{2}+\xi_{2}^{2} f_{5} \xi_{4} \xi_{1}+\xi_{2} f_{4} \xi_{4} \xi_{1}^{2}+2 \xi_{3} \xi_{2}^{3} f_{6} f_{4} \\
& +\xi_{3}^{2} \xi_{2}^{2} f_{6} f_{5}-4 \xi_{3}^{2} \xi_{1} f_{4} f_{6} \xi_{2} \\
& p_{3}=-2 f_{6}^{2} \xi_{1} \xi_{3}^{3}-\xi_{3}^{2} \xi_{1}^{2} f_{5}^{2}+2 \xi_{3}^{2} \xi_{1}^{2} f_{4} f_{6}-\xi_{3}^{2} \xi_{2} f_{6} f_{5} \xi_{1}+2 \xi_{3}^{2} f_{6}^{2} \xi_{2}^{2} \\
& +\xi_{3} \xi_{1}^{3} f_{5} f_{3}-2 \xi_{3} \xi_{1}^{3} f_{2} f_{6}-\xi_{3} \xi_{1}^{2} \xi_{2} f_{6} f_{3}-2 \xi_{3} \xi_{1}^{2} f_{6} \xi_{4}+2 \xi_{3} \xi_{1} \xi_{2}^{2} f_{5}^{2} \\
& -4 \xi_{3} \xi_{1} f_{4} f_{6} \xi_{2}^{2}+2 \xi_{3} f_{6} f_{5} \xi_{2}^{3}-\xi_{1}^{4} f_{1} f_{5}+2 \xi_{1}^{4} f_{4} f_{2}+2 \xi_{1}^{3} f_{4} \xi_{4}+2 \xi_{1}^{3} \xi_{2} f_{4} f_{3} \\
& -\xi_{1}^{3} \xi_{2} f_{6} f_{1}+\xi_{1}^{2} \xi_{2} f_{5} \xi_{4}+2 \xi_{1}^{2} f_{4}^{2} \xi_{2}^{2}+\xi_{1} \xi_{2}^{2} f_{6} \xi_{4}+2 \xi_{1} \xi_{2}^{3} f_{5} f_{4}+2 \xi_{2}^{4} f_{6} f_{4} \\
& p_{4}=\xi_{3}^{2} \xi_{1}^{2} f_{6} f_{5}-2 \xi_{3}^{2} \xi_{2} f_{6}^{2} \xi_{1}+\xi_{3} f_{3} f_{6} \xi_{1}^{3}-2 \xi_{3} \xi_{2} f_{5}^{2} \xi_{1}^{2}+2 \xi_{3} f_{4} f_{6} \xi_{2} \xi_{1}^{2} \\
& -2 \xi_{3} \xi_{2}^{2} f_{6} f_{5} \xi_{1}+2 \xi_{3} f_{6}^{2} \xi_{2}^{3}-f_{6} f_{1} \xi_{1}^{4}+2 f_{5} f_{2} \xi_{1}^{4}+2 f_{5} \xi_{4} \xi_{1}^{3}+2 \xi_{2} f_{5} f_{3} \xi_{1}^{3} \\
& +2 \xi_{2}^{2} f_{5} f_{4} \xi_{1}^{2}+\xi_{2} f_{6} \xi_{4} \xi_{1}^{2}+2 \xi_{2}^{3} f_{5}^{2} \xi_{1}+2 \xi_{2}^{4} f_{6} f_{5} \\
& p_{5}=2\left(f_{6} \xi_{1}^{2} \xi_{3}^{2}-\xi_{3} f_{5} \xi_{2} \xi_{1}^{2}-2 \xi_{3} f_{6} \xi_{2}^{2} \xi_{1}+f_{2} \xi_{1}^{4}+\xi_{1}^{3} \xi_{4}+\xi_{1}^{3} f_{3} \xi_{2}+f_{4} \xi_{2}^{2} \xi_{1}^{2}\right. \\
& \left.+\xi_{2}^{3} f_{5} \xi_{1}+f_{6} \xi_{2}^{4}\right) f_{6}
\end{aligned}
$$

Let the point $\left(p_{0}: \ldots: p_{5}\right)$ be represented by $P(X)=\sum_{i=0}^{5} p_{i} X^{i}$ and let

$$
g_{i}(X)=1-2 \frac{P_{i}(X)}{P_{i}\left(\theta_{i}\right)}, \quad i=1, \ldots, 6
$$


where $P_{i}(X)$ is defined by (3.1). We see that $g_{i}\left(\theta_{j}\right)=(-1)^{\delta_{i j}}$, so $g_{i}(X)^{2} \equiv 1$ $\bmod F(X)$. There are 6 commuting involutions $\varepsilon^{(i)}$ of $\mathcal{S}$, defined as follows:

$$
\varepsilon^{(i)}(P(X))=g_{i}(X) P(X) \quad \bmod F(X), \quad i=1, \ldots, 6 .
$$

In terms of coordinates $\pi_{j}$, one has

$$
\varepsilon^{(i)}\left(\pi_{j}\right)=(-1)^{\delta_{i j}} \pi_{j}
$$

Definition 3.4. We define $\operatorname{Inv}(\mathcal{S})$ to be the group of 32 commuting involutions of $S$ generated by the $\varepsilon^{(i)}$.

The $\mathbf{p}=\left(p_{0}: p_{1}: 0: 0: 0: 0\right)$ form a rational line $\Delta_{0} \subset \mathcal{S}$. We shall often write $p_{0}+p_{1} X \in \Delta_{0}$. Acting on $\Delta_{0}$ by the involutions gives 31 further lines.

Notation 3.5. We denote:

$$
\Delta_{i}=\varepsilon^{(i)}\left(\Delta_{0}\right), \quad \Delta_{i j}=\varepsilon^{(i)} \circ \varepsilon^{(j)}\left(\Delta_{0}\right) \quad \text { and } \quad \Delta_{i j k}=\varepsilon^{(i)} \circ \varepsilon^{(j)} \circ \varepsilon^{(k)}\left(\Delta_{0}\right) .
$$

The main result in this section describes how the nodes of $\mathcal{K}$ and $\mathcal{K}^{*}$ blow up.

Lemma 3.6. The map $\kappa$ blows up the node $N_{0}=(0: 0: 0: 1)$ of $\mathcal{K}$ into the line $\Delta_{0}$ and the 15 nodes $N_{i j}$ into the lines $\Delta_{i j}$. The tropes $T_{i}$ and $T_{i j k}$ blow up by $\kappa^{*}$ into the lines $\Delta_{i}$ and $\Delta_{i j k}$.

Proof. The node $N_{0}$ corresponds to the canonical class, so we consider divisors of the type $\mathfrak{X}=(x, y)+(u, v)$ with $u=x+h, h$ small and $v \approx-y \neq 0$. Then the local behavior of the Kummer coordinates is $\xi_{1}=1, \xi_{2}=2 x+h \approx 2 x, \xi_{3}=x(x+h) \approx x^{2}$ and

$$
\xi_{4}=\frac{F_{0}(x, x+h)-2 y v}{h^{2}} \approx \frac{4 y^{2}}{h^{2}} .
$$

Replacing this in the formulas for $\kappa$ and clearing denominators, then taking the limit as $h \rightarrow 0$ and dividing by $y^{4} \neq 0$, we obtain

$$
\begin{aligned}
\kappa(\xi) & \approx\left(-16 x y^{4}: 16 y^{4}: 0: 0: 0: 0\right) \\
& \approx(-x: 1: 0: 0: 0: 0) .
\end{aligned}
$$

Note that $\Delta_{0} \cap \Delta_{i}=\left(-\theta_{i}: 1: 0: 0: 0: 0\right)$, since for $\left(X-\theta_{i}\right) \in \Delta_{0}$ we have

$$
\varepsilon^{(i)}\left(X-\theta_{i}\right) \equiv g_{i}(X)\left(X-\theta_{i}\right) \equiv\left(X-\theta_{i}\right) \quad \bmod F(X) .
$$

We now show that $\Delta_{0} \cap \Delta_{i j}=\emptyset$ for $i \neq j$. Indeed, the intersection point $\mathfrak{p}$ should be invariant by $\varepsilon^{(i)} \circ \varepsilon^{(j)}$. A polynomial $P(X)$ represents such a point iff

$$
\begin{gathered}
\alpha P(X) \equiv g_{i}(X) g_{j}(X) P(X) \bmod F(X) \quad \text { for some } \alpha \in \bar{k}^{*} \\
F(X) \mid P(X)\left(\alpha-g_{i}(X) g_{j}(X)\right) .
\end{gathered}
$$

Replacing $X$ by the roots of $F(X)$ one sees that $P(X)$ must have at least two roots among $\theta_{k}$, so it must be of degree at least 2 and therefore cannot represent a point on $\Delta_{0}$. Similarly, $\Delta_{0} \cap \Delta_{i j k}=\emptyset$ for $i \neq j \neq k$.

The six $\Delta_{i}$ are strict transforms of the conics $\Gamma_{i}$ cut on $\mathcal{K}$ by the tropes $T_{i}$. To see this and to define $\kappa$ for points corresponding to divisors $\mathfrak{X}=\{x, y\}+\left\{\theta_{i}, 0\right\}$ with $y \neq 0$, write $F(X)=f_{6}\left(X-\theta_{i}\right) P_{i}(X)$. From this we get formulas for $f_{k}$, $k=0, \ldots, 6$ depending on $\theta_{i}$ and $h_{i j}, j=0, \ldots, 5$, the coefficients of $P_{i}(X)$, which we plug into $\xi_{4}=\left(F_{0}\left(x, \theta_{i}\right) /\left(x-\theta_{i}\right)^{2}\right)$. We substitute then $\xi_{1}=1, \xi_{2}=x+\theta_{i}$, 
$\xi_{3}=x \theta_{i}$ and $\xi_{4}$ in the formulas for $\kappa$. On multiplying by $\left(x-\theta_{i}\right)^{2} /\left(f_{6}^{2} P_{i}(x)\right)$ (note that $\left.P_{i}(x) \neq 0\right)$, we obtain

$$
P(X)=2\left(x-\theta_{i}\right) P_{i}(X)+P_{i}\left(\theta_{i}\right)(X-x),
$$

that is,

$$
\begin{aligned}
& p_{0}=2 h_{i 0}\left(x-\theta_{i}\right)-P_{i}\left(\theta_{i}\right) x, \\
& p_{1}=2 h_{i 1}\left(x-\theta_{i}\right)+P_{i}\left(\theta_{i}\right), \\
& p_{j}=2 h_{i j}\left(x-\theta_{i}\right) \quad \text { for } 2 \leq j \leq 5 .
\end{aligned}
$$

Equation (2.3) shows that the points $\left(1: x+\theta_{i}: x \theta_{i}: \xi_{4}\right)$ belong to the conic $\Gamma_{i}$. Formulas (3.9) give parametric equations (in $x$ ) of the strict transform of $\Gamma_{i}$ by $\kappa$. To confirm that this is $\Delta_{i}$, one verifies that

$$
P(X) \equiv P_{i}\left(\theta_{i}\right) g_{i}(X)(X-x) \quad \bmod F(X) .
$$

Applying the results in Section 4 and especially Corollary 4.12, one concludes that:

1) the tropes $T_{i}$ considered as singular points of $\mathcal{K}^{*}$, blow up by $\kappa^{*}$ into $\Delta_{i}$;

2) each of the fifteen $N_{i j}$ blows up into $\Delta_{i j}$;

3) the tropes $T_{i j k}, i \neq j \neq k$ blow up into $\Delta_{i j k}$;

4) the ten $\Delta_{i j k}(i \neq j \neq k)$ are strict transforms of the ten conics cut on $\mathcal{K}$ by the tropes (planes) not containing $N_{0}$. Each of them intersects six $\Delta_{i j}$ since each node is on six tropes.

\section{LiNe COMPLEXES}

Let $u=\left(u_{1}: u_{2}: u_{3}: u_{4}\right)$ and $v=\left(v_{1}: v_{2}: v_{3}: v_{4}\right)$ be distinct points in $\mathbb{P}^{3}$. Put $p_{i j}=u_{i} v_{j}-u_{j} v_{i}$. The Grassmann coordinates of the line $\langle u, v\rangle \subset \mathbb{P}^{3}$ are

$$
\mathfrak{p}=\left(p_{43}: p_{24}: p_{41}: p_{21}: p_{31}: p_{32}\right)=\left(X_{1}: \ldots: X_{6}\right) .
$$

The Grassmannian quadric $\mathcal{G} \subset \mathbb{P}^{5}$, representing the lines in $\mathbb{P}^{3}$ has the equation:

$$
G\left(X_{1}, \ldots, X_{6}\right)=2 X_{1} X_{4}+2 X_{2} X_{5}+2 X_{3} X_{6}=0 .
$$

Definition 4.1. A line complex of degree $d$ is a set of lines in $\mathbb{P}^{3}$ whose Grassmann coordinates satisfy a homogeneous equation $Q\left(X_{1}, \ldots, X_{6}\right)=0$ of degree $d$.

If $d=1$, this is called a linear complex, and if $d=2$, it is a quadratic complex.

A line $L \in \mathcal{G}$ parametrizes a pencil of lines in $\mathbb{P}^{3}$. The lines of a pencil $L$ all pass through a point $\mathfrak{f}(L)=u$, called the focus of the pencil, and lie in one plane $\mathfrak{h}(L)=\pi_{u}$, the plane of the pencil.

All lines in a linear complex $\mathcal{L}$ passing through a given point $u$ (respectively, lying in a plane $\pi$ ), form a pencil $L_{u}$ (respectively, $L_{\pi}$ ). Each linear complex $\mathcal{L}$ establishes a correspondence between points and planes in $\mathbb{P}^{3}$,

$$
I(u)=\mathfrak{h}\left(L_{u}\right), \quad I(\pi)=\mathfrak{f}\left(L_{\pi}\right), \quad I^{2}=1,
$$

which is also defined for lines; if $l \subset \mathbb{P}^{3}$ is the line $\left\langle u, u^{\prime}\right\rangle$, then $I(l)=I(u) \cap I\left(u^{\prime}\right)$. The line $I(l)$ is the polar line of $l$ with respect to the given linear complex.

Definition 4.2. Two linear complexes are called apolar if the correspondences they define commute. 
Let $H$ be any quadratic form in six variables such that the quadrics $G=0$ and $H=0$ intersect transversely, and denote by $\mathcal{H}=\left\{x \in \mathbb{P}^{5} \mid H(x)=0\right\}$. Let $\mathcal{W}=\mathcal{G} \cap \mathcal{H}$ and $\mathcal{A}=$ the set of lines on $\mathcal{W}$. The points in $\mathcal{W}$ represent the lines in $\mathbb{P}^{3}$ whose Grassmann coordinates $\mathfrak{p}$ satisfy $H(\mathfrak{p})=0$. A line $L \in \mathcal{A}$ represents a pencil of lines in $\mathbb{P}^{3}$ of the quadratic complex defined by $H$.

Definition 4.3. The Kummer surface $\mathcal{K} \subset \mathbb{P}^{3}$ associated to the quadratic complex $\mathcal{H}$ is the locus of focuses of such pencils: $\mathcal{K}=\{\mathfrak{f}(L) \mid L \in \mathcal{A}\}$.

Definition 4.4. The dual Kummer surface $\mathcal{K}^{*} \subset \mathbb{P}^{3^{\checkmark}}$ associated to the quadratic complex $\mathcal{H}$ is the locus of planes of such pencils.

From now on we suppose $f_{6}=1$.

Lemma 4.5. For any curve $\mathcal{C}$ of genus 2 , the Kummer surface belonging to the curve $\mathcal{C}$ given by (2.1) coincides with the Kummer surface just defined, if one takes the quadratic complex $\mathcal{H}$ to be given by

$$
\begin{aligned}
H= & -4 X_{1} X_{5}-4 X_{2} X_{6}-X_{3}^{2}+2 f_{5} X_{3} X_{6}+4 f_{0} X_{4}^{2} \\
& +4 f_{1} X_{4} X_{5}+4 f_{2} X_{5}^{2}+4 f_{3} X_{5} X_{6}+\left(4 f_{4}-f_{5}^{2}\right) X_{6}^{2} .
\end{aligned}
$$

Proof. See [3], Lemma 17.3.1 and pages 182-183.

If a point $\xi \in \mathbb{P}^{3}$ is the focus of the pencil corresponding to the line $L_{\xi} \in \mathcal{A}$, then $L_{\xi}$ lies in the plane $\Pi_{\xi} \subset \mathcal{G}$ corresponding to lines in $\mathbb{P}^{3}$ passing through $\xi$. But then the conic $\Pi_{\xi} \cap \mathcal{H}$ contains $L_{\xi}$, so it is degenerate; $\Pi_{\xi}$ is tangent to $\mathcal{H}$ and $\Pi_{\xi} \cap \mathcal{H}=L_{\xi} \cup L_{\xi}^{\prime}$. The lines of the quadratic complex passing through $\xi$ are in the two pencils $L_{\xi}$ and $L_{\xi}^{\prime}$, each with focus $\xi$, lying in the planes $\pi_{\xi}$ and $\pi_{\xi}^{\prime}$ in $\mathbb{P}^{3}$. The line $l_{\xi}=\pi_{\xi} \cap \pi_{\xi}^{\prime}$ is represented on $\mathcal{G}$ by the point $\mathfrak{p}_{\xi}=L_{\xi} \cap L_{\xi}^{\prime}$ and is called a singular line of the quadratic complex.

If $L_{\xi} \neq L_{\xi}^{\prime}$ the pencils are distinct and $\xi$ is a simple point of the Kummer; there is a one-to-one correspondence $\xi \leftrightarrow \mathfrak{p}_{\xi}$. However, if $L_{\xi}=L_{\xi}^{\prime}$, then $\pi_{\xi}=\pi_{\xi}^{\prime}$ and all the lines in $L_{\xi}$ are singular lines. The point $\xi$ is a singular point of the Kummer, because the map $\mathfrak{f}: \mathcal{A} \longrightarrow \mathcal{K}$ is algebraic. Therefore, the variety $\Sigma$ parametrizing singular lines is a desingularization of the Kummer.

Definition 4.6. The birational map $\kappa_{1}: \mathcal{K} \rightarrow \Sigma$ is defined by $\kappa_{1}(\xi)=\mathfrak{p}_{\xi}$.

Definition 4.7. The birational map $\kappa_{1}^{*}: \mathcal{K}^{*} \rightarrow \Sigma$ associates to a plane $\pi$ tangent to $\mathcal{K}$ the intersection point of the lines in $\mathcal{A}$ parametrizing the two pencils in $\mathcal{H}$ contained in $\pi$.

The maps $\kappa_{1}^{-1}$ and $\kappa_{1}^{*-1}$ extend to minimal desingularizations $\kappa_{1}^{-1}: \Sigma \longrightarrow \mathcal{K}$ and $\kappa_{1}^{*-1}: \Sigma \longrightarrow \mathcal{K}^{*}$ (see [3] and [6]). The following is proved in [3], page 181:

Lemma 4.8. The surface $\mathcal{K}^{*}$ is the projective dual of $\mathcal{K}$; that is, if $\xi=\mathfrak{f}(L) \in \mathcal{K}$, then $\eta=\mathfrak{h}(L) \in \mathcal{K}^{*}$ is the tangent plane of $\mathcal{K}$ at $\xi$. Therefore $\kappa_{1}(\xi)=\kappa_{1}^{*}(\eta)$.

Denote by $G(\vec{X}, \vec{Y})$ the bilinear form associated to the Grassmannian $G$. Make the change of coordinates

$$
\zeta_{i}=\frac{G\left(\vec{X}, \vec{v}\left(\theta_{i}\right)\right)}{\sqrt{\omega_{i}}}
$$


with vectors $\vec{v}\left(\theta_{i}\right)$ as in 3 formula (17.4.3). Let $\mathcal{K}$ be the Kummer surface associated to the quadratic complex $H$ of Lemma 4.5, By [6], Section 31 or [3] formula (17.4.2), a minimal desingularization of $\mathcal{K}$ is the $K 3$ surface in $\mathbb{P}^{5}$ :

$$
\Sigma=\Sigma_{0} \cap \Sigma_{1} \cap \Sigma_{2}, \quad \text { where } \quad \Sigma_{i}: \quad \sum_{j} \theta_{j}^{i} \zeta_{j}^{2}=0 \quad \text { for } \quad i=0,1,2 .
$$

Proposition 4.9. There is an explicit isomorphism $\Theta: \Sigma \longrightarrow \mathcal{S}$.

Proof. Let $\Theta: \Sigma \longrightarrow \mathcal{S}$ be defined by

$$
\Theta\left(\zeta_{1}: \ldots: \zeta_{6}\right)=\left(\frac{\zeta_{1}}{\sqrt{\omega_{1}}}: \ldots: \frac{\zeta_{6}}{\sqrt{\omega_{6}}}\right)=\left(\pi_{1}: \cdots: \pi_{6}\right) .
$$

To pass to variables $X_{j}$ and $p_{j}$, recall that $P(X)=\sum_{0}^{5} p_{j} X^{j}$. By (3.2) and (4.1):

$$
\frac{P\left(\theta_{i}\right)}{\omega_{i}}=\pi_{i}=\frac{\zeta_{i}}{\sqrt{\omega_{i}}}=\frac{G\left(\vec{X}, \vec{v}\left(\theta_{i}\right)\right)}{\omega_{i}} .
$$

Now, as polynomials in $X$, we have $G(\vec{X}, \vec{v}(X))=P(X)$, because both have degree 5 and agree on the six $\theta_{i}$. Explicit formulas for $\Theta$ are:

$$
\begin{array}{lll}
p_{0}=X_{1}+f_{1} X_{4} & p_{2}=X_{3}+2 f_{4} X_{5}+2 f_{3} X_{4}+f_{5} X_{6} & p_{4}=2 f_{5} X_{4}+2 X_{5} \\
p_{1}=X_{2}+2 f_{2} X_{4}+f_{3} X_{5} & p_{3}=2 f_{4} X_{4}+2 f_{5} X_{5}+2 X_{6} & p_{5}=2 X_{4} .
\end{array}
$$

Proposition 4.10. Denoting by $\kappa^{-1}$ and $\kappa_{1}^{-1}$ the blow-downs from $\mathcal{S}$, respectively, $\Sigma$ to $\mathcal{K}$ one has $\kappa_{1}^{-1}=\kappa^{-1} \circ \Theta$.

Proof. Pick a point $\xi \in \mathbb{P}^{3}$ and write the equations of the plane $\Pi_{\xi} \subset \mathcal{G}$ of lines through $\xi$ (see equations (4.7) of [8]). Take $\mathcal{H}$ to be defined as in Lemma 4.5. Now, $\Pi_{\xi}$ is tangent to $\mathcal{H}$ iff the intersection consists of two lines: $\Pi_{\xi} \cap \mathcal{H}=L_{\xi} \cup L_{\xi}^{\prime}$. Computing in terms of $\xi$ the coordinates of $\mathfrak{p}_{\xi}=L_{\xi} \cap L_{\xi}^{\prime}$, we find homogeneous formulas for $X_{i}$ in $\xi_{i}$ of degree $4: \mathfrak{p}_{\xi}=\left(X_{1}(\xi): \ldots: X_{6}(\xi)\right)=\kappa_{1}(\xi)$. Comparing $\kappa(\xi)=\left(p_{0}(\xi): \ldots: p_{5}(\xi)\right)$ from Lemma 3.3 with

$$
\Theta \circ \kappa_{1}(\xi)=\left(\hat{p}_{0}(\xi): \cdots: \hat{p}_{5}(\xi)\right): \mathcal{K} \longrightarrow \mathcal{S}
$$

yields $\hat{p}_{i} p_{5}-\hat{p}_{5} p_{i}=\delta_{i} K$, with $K$ given by (2.2), for $\delta_{i}$ a homogeneous polynomial in $\xi$.

Associated with a quadratic complex $\mathcal{H}: H=0$ there is a set of 6 mutually apolar linear complexes $\mathcal{L}_{k}$, such that the polar of any line in $\mathcal{H}$ with respect to $\mathcal{L}_{k}$ is in $\mathcal{H}$. If $G$ and $H$ are written in diagonal form, these complexes are $\mathcal{L}_{k}: \zeta_{k}=0$ for $k=1, \ldots, 6$. The action of the correspondences $I_{k}$ on lines in $\mathbb{P}^{3}$ translates in coordinates $\zeta=\left(\zeta_{1}: \ldots: \zeta_{6}\right)$ by

$$
I_{k}\left(\zeta_{i}\right)=(-1)^{\delta_{i k}} \zeta_{i}
$$

which restricts to $\Sigma$. The Kummer surface is determined by $\mathcal{H}$, so it is invariant under the transformation $I_{k}$. The set of nodes and tropes is invariant (see [6], Section 30).

Proposition 4.11. Let $W_{k}$ be as in Lemma 2.4. For any $k$, the map $I_{k}$ is the unique automophism of $\Sigma$ such that $\kappa_{1}^{*-1} \circ I_{k}=W_{k} \circ \kappa_{1}^{-1}$. 
Proof. Let $\xi \in \mathcal{K}$ be a simple point and denote $\mathfrak{p}_{\xi}=\kappa_{1}(\xi)$. For a subset $V \subset$ $\mathcal{G}$, put $I_{k}(V)=\left\{I_{k}(l) \in \mathcal{G} \mid l \in V\right\}$. The pencils $I_{k}\left(L_{\xi}\right)$ and $I_{k}\left(L_{\xi}^{\prime}\right)$ are both contained in the polar plane of $\xi$ with respect to $\mathcal{L}_{k}$, which by Lemma 4.13 is $W_{k}(\xi)$. The plane in $\mathbb{P}^{5}$ parametrizing lines in $W_{k}(\xi)$ is therefore tangent to $\mathcal{H}$ at $I_{k}\left(L_{\xi}\right) \cap I_{k}\left(L_{\xi}^{\prime}\right)=I_{k}\left(L_{\xi} \cap L_{\xi}^{\prime}\right)=I_{k}\left(\mathfrak{p}_{\xi}\right)=I_{k} \circ \kappa_{1}(\xi)$. By definition of $\kappa_{1}^{*}$ we have $\kappa_{1}^{*}\left(W_{k}(\xi)\right)=I_{k} \circ \kappa_{1}(\xi)$.

The following corollary illustrates how the projective duality (over $k\left(\theta_{k}\right)$ ) between $\mathcal{K}$ and $\mathcal{K}^{*}$ lifts to $\mathcal{S}$.

Corollary 4.12. For any $k$, the map $\varepsilon^{(k)}$ is the unique automophism of $\mathcal{S}$ such that $\kappa^{*-1} \circ \varepsilon^{(k)}=W_{k} \circ \kappa^{-1}$.

Proof. Let $\xi \in \mathcal{K}$ and $\eta \in \mathcal{K}^{*}$ be dual. We have:

$$
\Theta \circ \kappa_{1}^{*}(\eta) \stackrel{(4.8)}{=} \Theta \circ \kappa_{1}(\xi) \stackrel{(4.10)}{=} \kappa(\xi) \stackrel{(3.2)}{=} \kappa^{*}(\eta) .
$$

Note that $\Theta \circ I_{k} \circ \Theta^{-1}=\varepsilon^{(k)}$, by (4.2), (4.3) and (3.7). Therefore,

$$
\kappa^{*-1} \circ \varepsilon^{(k)} \stackrel{4.4}{=} \kappa_{1}^{*-1} \circ \Theta^{-1} \circ \Theta \circ I_{k} \circ \Theta^{-1} \stackrel{4.11}{=} W_{k} \circ \kappa_{1}^{-1} \circ \Theta^{-1} \stackrel{4.10)}{=} W_{k} \circ \kappa^{-1} \text {. }
$$

This is summarized in the following diagram, where the arrows to $\mathcal{K}$ and $\mathcal{K}^{*}$ are the minimal desingularizations:

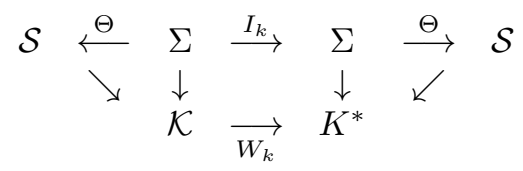

Now Corollary 4.12 is useful for finding explicit formulas for $\kappa^{*}$, because

$$
\kappa^{*}=\kappa^{*} \circ W_{i} \circ \kappa^{-1} \circ \kappa \circ W_{i}^{-1}=\varepsilon^{(i)} \circ \kappa \circ W_{i}^{-1}
$$

on an open dense set in $\mathcal{K}^{*}$. We find formulas for $W_{i}$ in [8], Lemma 4.13. The resulting formulas for $\kappa^{*}$ are huge and not listed in this paper, since on a given example it is easier to apply successively each map involved.

Lemma 4.13. For any point $\xi \in \mathbb{P}^{3}$ the plane with dual coordinates $W_{i}(\xi)$ is the polar plane of $\xi$ with respect to $\mathcal{L}_{i}$.

Proof. See [8, Lemma 4.14.

\section{Linear aUtomorphisms of $\mathcal{S}$}

Keeping Notation 3.5, we let

$$
\begin{aligned}
\mathfrak{p}_{i} & =\Delta_{0} \cap \Delta_{i}, \\
\mathfrak{p}_{i j} & =\Delta_{i} \cap \Delta_{i j}=\varepsilon^{(i)}\left(\mathfrak{p}_{j}\right), \\
\mathfrak{p}_{i j k} & =\Delta_{i j} \cap \Delta_{i j k}=\varepsilon^{(i)}\left(\mathfrak{p}_{j k}\right) .
\end{aligned}
$$

There are no other lines on $\mathcal{S}([5]$, page 775$)$, so this is the whole structure of line intersections on $\mathcal{S}$. Now let $\mathrm{GL}(\mathcal{S})$ be the group of linear automorphisms of $\mathcal{S}$.

Lemma 5.1. Let $A, B \in G L(\mathcal{S})$ such that $A_{\left.\right|_{\Delta_{0}}}=B_{\left.\right|_{\Delta_{0}}}$. Then $A=B$. 
Proof. Let $I \in \mathrm{GL}(\mathcal{S})$ be the identity. If $A \in \mathrm{GL}(\mathcal{S})$ and $A_{\left.\right|_{\Delta_{0}}}=I_{\left.\right|_{\Delta_{0}}}$, then $A$ fixes the $\mathfrak{p}_{i}$, so invaries the $\Delta_{i}$. But then by $A$ invaries also $\Delta_{i j}$, the unique line other than $\Delta_{0}$ which meets $\Delta_{i}$ and $\Delta_{j}$, so $A$ fixes $\mathfrak{p}_{i j}, j=1, \ldots, 6$. Hence $A_{\left.\right|_{\Delta_{i}}}=I_{\left.\right|_{\Delta_{i}}}$. Similarly, one sees that $A$ is the identity on any of the 32 lines on $\mathcal{S}$, so $A=I$.

Let $A \in \operatorname{GL}(\mathcal{S})$. Since $A\left(\Delta_{0}\right)$ is a line, by Lemma 5.1 there exists a unique involution $\varepsilon \in \operatorname{Inv}(\mathcal{S})$ such that $\varepsilon \circ A\left(\Delta_{0}\right)=\Delta_{0}$. We associate to $A$ the permutation $\sigma \in S_{6}$ such that

$$
\varepsilon \circ A\left(\mathfrak{p}_{i}\right)=\mathfrak{p}_{\sigma(i)} \quad \text { for } i=1, \ldots, 6 .
$$

Note that $\sigma=$ id iff $\varepsilon \circ A_{\left.\right|_{\Delta_{0}}}=I_{\left.\right|_{\Delta_{0}}}$ iff $\varepsilon \circ A=I$ (by Lemma 5.1) iff $A \in \operatorname{Inv}(\mathcal{S})$.

Definition 5.2. $\mathrm{GL}_{0}(\mathcal{S})$ is the subgroup of $\mathrm{GL}(\mathcal{S})$ of linear automorphisms $A$ such that $A\left(\Delta_{0}\right)=\Delta_{0}$.

Lemma 5.3. Let $A \in G L(\mathcal{S})$ and $\sigma \in S_{6}$ be the permutation associated to $A$ by (5.2). Then, for any $1 \leq i \leq 6$ we have:

$$
A \circ \varepsilon^{(i)}=\varepsilon^{(\sigma(i))} \circ A .
$$

Proof. Let $B=\varepsilon \circ A$. Then $B\left(\Delta_{0}\right)=\Delta_{0}$ and $B\left(\mathfrak{p}_{i}\right)=\mathfrak{p}_{\sigma(i)}$, so $B\left(\Delta_{i}\right)=\Delta_{\sigma(i)}$. The unique line cutting $\Delta_{\sigma(i)}$ and $\Delta_{\sigma(j)}$ is $\Delta_{\sigma(i) \sigma(j)}$, hence $B\left(\Delta_{i j}\right)=\Delta_{\sigma(i) \sigma(j)}$. Then

$$
B\left(\mathfrak{p}_{i j}\right)=B\left(\Delta_{i} \cap \Delta_{i j}\right)=B\left(\Delta_{i}\right) \cap B\left(\Delta_{i j}\right)=\Delta_{\sigma(i)} \cap \Delta_{\sigma(i) \sigma(j)}=\mathfrak{p}_{\sigma(i) \sigma(j)} .
$$

Now one sees that $(\varepsilon \circ A)^{-1} \circ \varepsilon^{(\sigma(i))} \circ(\varepsilon \circ A)$ acts like $\varepsilon^{(i)}$ on $\mathfrak{p}_{j}$. By Lemma 5.1 and knowing that $\operatorname{Inv}(\mathcal{S})$ is commutative, we conclude $A \circ \varepsilon^{(i)}=\varepsilon^{(\sigma(i))} \circ A$.

Proposition 5.4. Let $\psi: G L(\mathcal{S}) \longrightarrow G L_{0}(\mathcal{S})$ be the map $A \mapsto \varepsilon \circ A$ defined by formula (5.2). We have an exact sequence of groups

$$
1 \longrightarrow \operatorname{Inv}(\mathcal{S}) \longrightarrow G L(\mathcal{S}) \stackrel{\psi}{\longrightarrow} G L_{0}(\mathcal{S}) \longrightarrow 1 .
$$

By Proposition [5.4, $\operatorname{Inv}(\mathcal{S})=\operatorname{ker}(\psi)$ is a normal subgroup of $\mathrm{GL}(\mathcal{S})$.

Corollary 5.5. For any linear automorphism $A$ of $\mathcal{S}$ not in $\operatorname{Inv}(\mathcal{S})$, the centralizer of $A$ in $\operatorname{Inv}(\mathcal{S})$ is not equal to $\operatorname{Inv}(\mathcal{S})$.

We now show that $\mathrm{GL}_{0}(\mathcal{S})$ is in bijection with the group of linear automorphisms of $\Delta_{0}$ which invariate the set $\left\{p_{i}, i=1, \ldots, 6\right\}$. Lemma 5.1 and Proposition 5.6 below give necessary and sufficient conditions for the existence of nontrivial elements of $\mathrm{GL}_{0}(\mathcal{S})$. For the existence of noncommuting involutions of $\mathcal{S}$, see [8, Section 6 .

Proposition 5.6. Let $\sigma \in S_{6}$ and let $B: \Delta_{0} \longrightarrow \Delta_{0}$ be a linear automorphism of $\Delta_{0}$ such that for $1 \leq i \leq 6$, we have $B\left(\mathfrak{p}_{i}\right)=\mathfrak{p}_{\sigma(i)}$. Then there exists a unique $A \in G L_{0}(\mathcal{S})$ such that $A_{\left.\right|_{\Delta_{0}}}=B$.

Proof. Suppose $\sigma$ and $B$ given. If $A$ exists, it is unique by Lemma 5.1 and $\sigma$ is the permutation associated to $A$ defined by (5.2). Let $\widetilde{A}$ be the linear operator of $\mathcal{P}_{5}$ (polynomials of degree $\leq 5$ ) associated to $A$. Let $a, b, c, d \in \bar{k}$ such that

$$
\widetilde{A}(1)=a X+b \quad \text { and } \quad \widetilde{A}(X)=c X+d .
$$


After some linear algebra and using (5.3), we find that the image of a point $\mathfrak{p} \in \mathcal{S}$ represented by $P(X)=\sum_{j} \pi_{j} P_{j}(X)$ is

$$
\widetilde{A}(P(X))=\sum_{j} \underbrace{\left(\pi_{j} \frac{\omega_{j}}{\omega_{\sigma(j)}}\left(a \theta_{\sigma(j)}+b\right)\right)}_{\pi_{\sigma(j)}^{\prime}} P_{\sigma(j)}(X) .
$$

We have to prove that the point $\left(\pi_{1}^{\prime}: \ldots: \pi_{6}^{\prime}\right)$ satisfies equations (3.3).

We show that $k_{\sigma(j)} \omega_{\sigma(j)}{\pi^{\prime}}_{\sigma(j)}^{2}=\alpha_{j} \omega_{j} \pi_{j}^{2}$ for a quadratic polynomial $\alpha_{j}$ in $\theta_{j}$. We have:

and then

$$
k_{\sigma(j)} \omega_{\sigma(j)} \pi_{\sigma(j)}^{\prime 2}=k_{\sigma(j)}\left(a \theta_{\sigma(j)}+b\right)^{2} \frac{\omega_{j}}{\omega_{\sigma(j)}} \omega_{j} \pi_{j}^{2},
$$

$$
\alpha_{j}=k_{\sigma(j)}\left(a \theta_{\sigma(j)}+b\right)^{2} \frac{\omega_{j}}{\omega_{\sigma(j)}} .
$$

Write $\widetilde{A}\left(X-\theta_{i}\right)$ in two ways, using the fact that $A\left(\mathfrak{p}_{i}\right)=\mathfrak{p}_{\sigma(i)}$ or linearity of $\widetilde{A}$ :

$$
\mu_{j}\left(X-\theta_{\sigma(j)}\right)=\widetilde{A}\left(X-\theta_{j}\right)=c X+d-\theta_{j}(a X+b) \quad \text { where } \mu_{j} \in \bar{k} .
$$

Replacing $X=\theta_{\sigma(j)}$, we obtain the formula

$$
\theta_{j}=\frac{c \theta_{\sigma(j)}+d}{a \theta_{\sigma(j)}+b},
$$

which gives the relations between the roots of $F(X)$ necessary for the existence of the linear automorphism $B$.

Now, we calculate $\omega_{j}$ replacing each $\theta_{j}$ by the formula (5.5):

$$
\begin{aligned}
\omega_{j} & =\prod_{i \neq j}\left(\theta_{i}-\theta_{j}\right)=\prod_{i \neq j}\left(\frac{c \theta_{\sigma(i)}+d}{a \theta_{\sigma(i)}+b}-\frac{c \theta_{\sigma(j)}+d}{a \theta_{\sigma(j)}+b}\right) \\
& =\frac{1}{\left(a \theta_{\sigma(j)}+b\right)^{4}} \underbrace{\frac{1}{\prod_{i}\left(a \theta_{\sigma(i)}+b\right)}}_{\text {constant }} \prod_{i \neq j}(\left(\theta_{\sigma(i)}-\theta_{\sigma(j)}\right) \underbrace{(b c-a d)}_{\text {constant }}) .
\end{aligned}
$$

Call $\gamma$ the constant part of the equation:

$$
\frac{\omega_{j}}{\omega_{\sigma(j)}}=\frac{\gamma}{\left(a \theta_{\sigma(j)}+b\right)^{4}} .
$$

Replacing (5.6) in $\alpha_{j}$, we have:

$$
\alpha_{j}=k_{\sigma(j)}\left(a \theta_{\sigma(j)}+b\right)^{2} \frac{\gamma}{\left(a \theta_{\sigma(j)}+b\right)^{4}}=\gamma \frac{k_{\sigma(j)}}{\left(a \theta_{\sigma(j)}+b\right)^{2}} .
$$

To see that $\alpha_{j}$ is quadratic in $\theta_{j}$ (for each $k_{j}$ ), we use formula (5.5) to obtain:

$$
\begin{aligned}
a \theta_{j}-c & =\frac{a d-b c}{a \theta_{\sigma(j)}+b} \quad \text { which gives the result for } k_{\sigma(j)}=1 ; \\
a^{2} \theta_{j}^{2}-c^{2} & =\frac{2 a c(a d-b c) \theta_{\sigma(j)}+a^{2} d^{2}-b^{2} c^{2}}{\left(a \theta_{\sigma(j)}+b\right)^{2}} \\
\text { which gives the result for } k_{\sigma(j)} & =\theta_{\sigma(j)} ; \\
b \theta_{j}-d & =\frac{(b c-a d) \theta_{\sigma(j)}}{a \theta_{\sigma(j)}+b} \quad \text { which gives the result for } k_{\sigma(j)}=\theta_{\sigma(j)}^{2} .
\end{aligned}
$$




\section{REFERENCES}

[1] A. Beauville, Surfaces algébriques complexes. Soc. Math. France, 1978. MR0485887 (58:5686)

[2] P. Corn, Tate-Shafarevich Groups and K3-Surfaces, Math. Comp. 79 (2010), 563-581. MR.2552241(2011d:11139)

[3] J.W.S. Cassels and E.V. Flynn, Prolegomena to a Middlebrow Arithmetic of Curves of Genus 2. London Math. Soc. Lecture Note Series 230, Cambridge, 1996. MR 1406090 (97i:11071)

[4] E. V. Flynn, The Jacobian and formal group of a curve of genus 2 over an arbitrary ground field, Math. Proc. Cambridge Phil. Soc. 107 (1990), 425-441. MR.1041476 (91b:14025)

[5] Griffiths and Harris, Principles of Algebraic Geometry. John Wiley and Sons, New York, 1978. MR507725 (80b:14001)

[6] R.W.H.T. Hudson, Kummer's Quartic Surface. Cambridge Univ. Press, 1990. MR1097176 (92e:14033)

[7] A. Logan and R. van Luijk, Nontrivial elements of Sha explained through K3 surfaces, Math. Comp. 78 (2009), 441-483. MR2448716 (2010a:14048)

[8] V.G. Lopez-Neumann and C. Manoil, Explicit computations on the desingularized Kummer surface, arXiv:0906.0790v1.

Faculdade de Matemática, Universidade Federal de Uberlândia, MG, Brazil

E-mail address: gonzalo@famat.ufu.br

Section de Mathématiques, Université de Genève, CP 64, 1211 Geneva 4, Switzerland E-mail address: constantin.manoil@edu.ge.ch

Current address: Collège Sismondi, 3 Chemin Rigot, 1202 Genève (Geneva), Switzerland 